\title{
Bronchoscopic evaluation of children less than 5 years old with stridor
}

\author{
Kaustubh Mohite ${ }^{1}$ and Mahesh Mohite $^{2}$ \\ ${ }^{1}$ Narayana Health \\ ${ }^{2}$ Bai Jerbai Wadia Hospital for Children
}

May 13, 2021

\begin{abstract}
Background: Stridor is a harsh, vibratory sound with variable pitch which is caused by partial obstruction of airway that results in turbulent airflow. It is mainly due to anatomic deformities in the infantile age group. However, various acquired conditions affecting upper airway may also cause stridor. Here, we evaluated 87 cases of stridor using Flexible bronchoscope in order to determine the cause of stridor. Objective: To determine the clinical characteristics, Flexible bronchoscopy findings and clinical correlation in children admitted in our hospital with complains of stridor. Design: Retrospective observational study. Setting: Single center study conducted in Amrut medical foundation. 87 children aged less than 5 years with a clinical complains of stridor were included in the study and Flexible bronchoscopy was performed. The findings were analyzed and results were reported. Results: A total of 87 children with stridor were enrolled in the study and Flexible Bronchoscopy was performed in them. Of these, 68 children had an abnormal bronchoscopy finding. Laryngomalacia was the commonest cause of stridor observed followed by subglottic stenosis and tracheomalacia. Cough was the commonest presenting symptom associated with stridor and tachypnea was the commonest clinical sign observed in these children. Conclusion: Flexible bronchoscopy plays an important role in diagnosing the exact cause of stridor in pediatric age group. Key words: Stridor, Flexible Bronchoscopy, Laryngomalacia.
\end{abstract}

Title Page

Type of the article: Brief Report

Title of the Article: Bronchoscopic evaluation of children less than 5 years old with stridor Author Details:

\begin{tabular}{|c|c|c|c|c|c|c|c|}
\hline $\begin{array}{l}\text { Author } \\
\text { Order }\end{array}$ & $\begin{array}{l}\text { First name } \\
\text { (Full name } \\
\text { to be } \\
\text { provided) }\end{array}$ & $\begin{array}{l}\text { Middle } \\
\text { name }\end{array}$ & $\begin{array}{l}\text { Last name } \\
\text { (Full name } \\
\text { to be } \\
\text { provided) }\end{array}$ & $\begin{array}{l}\text { Designation } \\
\text { /Post held }\end{array}$ & Department & $\begin{array}{l}\text { Institute } \\
\text { Name }\end{array}$ & $\begin{array}{l}\text { Emails/ } \\
\text { Other } \\
\text { Details }\end{array}$ \\
\hline No. 1 & $\begin{array}{l}\text { Dr } \\
\text { Kaustubh }\end{array}$ & & Mohite & $\begin{array}{l}\text { M.D. } \\
\text { Pediatrics }\end{array}$ & Pediatrics & $\begin{array}{l}\text { Narayana } \\
\text { Health, } \\
\text { Pediatrics }\end{array}$ & drkaustubhm \\
\hline No. 2 & $\begin{array}{l}\text { Dr } \\
\text { Mahesh }\end{array}$ & & Mohite & $\begin{array}{l}\text { M.D } \\
\text { Pediatrics }\end{array}$ & Pediatrics & $\begin{array}{l}\text { Bai Jerbai } \\
\text { Wadia } \\
\text { Hospital } \\
\text { for } \\
\text { Children }\end{array}$ & mahesh.mohit \\
\hline
\end{tabular}


- Corresponding Author Details:Dr Kaustubh Mohite Fellow in Pediatric Pulmonology, Narayana Health City, Bangalore - 560099Email ID- drkaustubhmohite@gmail.com

- Contributors Details: KM and MM conceptualized the study. Data collection done by KM. Analysis done by KM and MM. Article reviewed by KM and MM and approved by all authors.

- Key words: Flexible Bronchoscopy, Stridor, Laryngomalacia

- Running Title of the article: Bronchoscopic Evaluation of children with stridor.

- Abbreviated Title of the article: Bronchoscopic Evaluation of children with stridor.

- Funding: None

- Conflict of interest: None

- Acknowledgement: We would like to express our gratitude towards the patients and their parents for being a part of this study.

\section{Introduction:}

Stridor is a sign of upper airway obstruction. It may be inspiratory, expiratory or biphasic depending on the site of obstruction. Inspiratory stridor suggests airway obstruction above the glottis while lower tracheal lesion presents with expiratory stridor. A biphasic stridor suggests a glottic or subglottic lesion. Stridor may be caused by abnormalities in nose, pharynx, larynx or trachea. Laryngomalacia stays the commonest cause of stridor amongst infants (1). Other causes include various congenital and acquired conditions like subglottic stenosis, tracheal stenosis, gastro-esophageal reflux disease (GERD), foreign body aspiration, vocal cord paralysis, external tracheal obstruction, laryngeal web, hemangioma and cysts $(2,3)$. It is essential to determine the appropriate etiology of stridor in order to start prompt therapy.

Flexible bronchoscopy (FB) plays an important role as a diagnostic modality to determine the etiology of stridor. It helps in diagnosing both static anatomic or dynamic obstruction in upper and lower airway of children. FB is not a routine investigation carried out in all children with stridor; however, if done in cases with chronic and severe cases, can provide a prompt diagnosis. During evaluation of upper airway to find for the cause of stridor, it is advisable to evaluate the lower airway too as almost $68 \%$ of the patients with upper airway anomalies have associated lower airway anomalies (4).

This study aims to describe the clinical characteristics, FB findings and its clinical correlation in children having clinical symptoms of stridor.

\section{Materials \& Methods:}

This is a single center retrospective observational study conducted in Amrut Medical foundation, Panvel, Maharashtra. All children less than 5 years old with clinical complains of stridor admitted in our hospital were enrolled in the study. FB was done in children in all these patients having stridor with or without other respiratory findings like wheezing, respiratory distress and cyanosis. Demographic characteristics, symptoms, physical examination on presentation, FB findings, presence of associated co-morbidities were obtained from the patients' medical records. All these findings were assembled in a tabular format and the data was analyzed.

FB was performed by the same pulmonologist with a flexible bronchoscope (Pentax videoscope 3.5 with working port $1.2 \mathrm{~mm}$ ). The procedure was explained to the parents of the selected children and consent was taken. Before every procedure, both the nasal cavities were examined and the wider nostril was selected for introduction of FB. 2\% Lignocaine jelly was used as a topical anesthetic agent which was introduced in the nasal cavity before the procedure. The child was given intravenous sedation with IV Propofol or IV Midazolam before initiation of the procedure. All emergency drugs and equipments were kept bedside. The child was continuously monitored for heart rate, respiratory rate, pulse oximetry and non-invasive blood pressure during the procedure. Continuous Oxygen was delivered via nasal cannula in order to keep $\mathrm{SpO}_{2}$ above $92 \%$.

FB was performed through trans-nasal approach while the patient was breathing spontaneously and under conscious sedation with the above-mentioned drugs. The anatomy of nose, nasopharynx and larynx were 
noted. Active movement of the vocal cords were observed. After completing the evaluation of the vocal cord movement, injection $2 \%$ lignocaine was flushed through the port of FB over the vocal cords as local anesthesia. Gradually the decreased movement of vocal cord was observed and then the scope was negotiated into the trachea to observer the abnormalities of trachea-bronchial tree.

\section{Statistical Analysis:}

Data entry was done in Microsoft Excel and was statistically analyzed using SPSS software. Descriptive statistical data like means, medians and standard deviations were calculated for continuous variables.

\section{Results:}

A total of 87 children less than 5 years old with stridor were involved in the study. FB was done in all these selected subjects. The mean age was 20 months and 13 days with the youngest being 3 days old and eldest being 5 years. Amongst these, $53(60.9 \%)$ were boys and $34(39.1 \%)$ were girls. The most common symptom associated with stridor was cough which was observed in $45(51.72 \%)$ children followed by breathlessness $(32.18 \%)$ and fever $(18.39 \%)$. There were $4(4.6 \%)$ children who presented with severe respiratory distress involving peripheral and central cyanosis along with grunting. On examination, the most common finding associated with stridor was tachypnea which was observed in $74(85.05 \%)$ cases. Based on the clinical history and examination, 21 (24.13\%) children were suspected to have laryngomalacia.

FB was done under all aseptic precautions as mentioned above and the observations were analyzed. 68 (78.16\%) out of 87 children with clinical stridor had an abnormal FB finding. Out of these, laryngomalacia was the most common abnormality noted amounting to 20 (29.41\%) followed by subglottic stenosis $(13.23 \%)$ and tracheomalacia (11.76\%). Other abnormalities included foreign body aspiration, external compression of airway, laryngeal edema, cyst, GERD, congenital web and dynamic tracheal obstruction. Out of the 21 clinically suspected cases of laryngomalacia, 20 (95.23\%) cases were observed to have laryngomalacia on FB. Of all these cases, $78(89.65 \%)$ children were managed conservatively while $9(10.35 \%)$ were referred for surgical intervention. These cases involved severe laryngomalacia causing significant respiratory distress, foreign body aspiration, congenital web and airway cyst. There were no adverse events in any of the cases during the procedure.

\section{Discussion:}

Stridor is one of the common respiratory finding observed in pediatric age group which is caused mostly by some obstruction in the upper airway. Stridor is not exclusively present in respiratory distress but could also have some central nervous system etiology. In the first year of life, Laryngomalacia is the most common cause of stridor (1). It presents typically in the first few weeks after birth, may worsen in the first 4 - 8 months and usually resolves by $18-20$ months of age (4). It is usually managed conservatively; but surgical management is necessary in cases with worsening respiratory distress (5). However, secondary airway lesions other than laryngomalacia may also be observed to cause stridor in pediatric age group. FB plays an important role in determining these causes of airway anomalies.

Various studies were performed worldwide to evaluate the cause of stridor in this age group by bronchoscopic evaluation $(6,7,8)$. Most of the studies had laryngomalacia as the most common cause of stridor with an associated secondary lower airway anomaly. Laryngomalacia may be caused by multiple reasons. One of the most common cause of laryngomalacia is neurological, i.e. altered laryngeal tone due to abnormality of the laryngeal nerve (9). Reflux is said to be another important cause of irritation and edema of the supra-glottic airway which worsens airway obstruction (10). Laryngomalacia is mainly a clinical diagnosis having stridor which worsens with feeding and in supine position and decreases on prone positioning. Other associated features involve weight loss of failure to thrive. Infants with laryngomalacia are observed to have shortened aryepiglottic folds, floppy omega shaped epiglottis and redundant arytenoid tissue which prolapses over the glottis in every breathing cycle. In our study, there was a good correlation (95.23\%) between clinical diagnosis and bronchoscopic findings in cases with suspicion of Laryngomalacia.

There may be other causes of stridor beyond laryngomalacia. In a study conducted by Martin et al, infra- 
glottic stenosis, laryngeal inflammation and vocal cord paralysis were the most common findings on bronchoscopy in children with stridor (11). In other study by Erdem et al, laryngomalacia was the most common cause for stridor followed by other causes like subglottic stenosis, tracheal compression, laryngeal cyst which comprised of $16.5 \%$ of the cases evaluated for stridor (1). In our study, laryngomalacia was the most common cause of stridor (29.41\%) followed by subglottic stenosis $(13.23 \%)$ and tracheomalacia (11.76\%). Other findings included subglottic stenosis, tracheomalacia, foreign body aspiration, external airway compression etc. Subglottic stenosis involves narrowing of the airway encircled by the cricoid cartilage, below the glottis and above the first tracheal ring (12). It could be congenital or acquired. More than $90 \%$ of the acquired subglottic stenosis is secondary to endotracheal intubation (13). Foreign body aspiration is a very common cause of acute onset noisy breathing in pediatric age group. Classic history of acute onset choking or coughing might not be present in such cases. In the above study done by Erdem et al, $15 \%$ of the children with stridor required surgical intervention (1) as compared to $10.35 \%$ of the children requiring surgical intervention in our study.

Stridor improved with time in all cases as observed on follow up. Children with an underlying neurological ailment tend to have persistent stridor for a relatively longer duration (1). However, Yuen et al reported in his study that neurological abnormalities did not alter time to resolution of symptoms (14).

On the basis of various studies done in the past, it can be concluded that bronchoscopic evaluation is necessary in accurately diagnosing the exact cause of stridor as prompt treatment could be initiated. Pediatric bronchoscopy can be done by a trained pulmonologist, intensivist, surgeon or anesthetist and should be included among the primary investigations in evaluation of children with stridor.

\section{Reference:}

1. Erdem E, Gokdemir Y, Unal F, Ersu R, Karadag B, Karakoc F. Flexible bronchoscopy as a valuable tool in the evaluation of infants with stridor. European Archives of Oto-Rhino-Laryngology. 2013 Jan;270(1):21-5.

2. Holinger LD. Congenital Laryngeal anomalies, Pediatric Laryngology and Bronchoesophagology, ED by Holinger, LD, Lusk, RP, Green, CG.

3. HOLZKI J, LASCHAT M, STRATMANN C. Stridor in the neonate and infant. Implications for the paediatric anaesthetist. Prospective description of 155 patients with congenital and acquired stridor in early infancy. Pediatric Anesthesia. 1998 May;8(3):221-7.

4. Nussbaum E, Maggi JC. Laryngomalacia in children. Chest. 1990 Oct 1;98(4):942-4.

5. Mancuso RF, Choi SS, Zalzal GH, Grundfast KM. Laryngomalacia: the search for the second lesion. Archives of Otolaryngology-Head \& Neck Surgery. 1996 Mar 1;122(3):302-6.

6. Vijayasekaran D, Gowrishankar NC, Kalpana S, Vivekanandan VE, Balakrishnan MS, Suresh S. Lower airway anomalies in infants with laryngomalacia. The Indian Journal of Pediatrics. 2010 Apr;77(4):4036.

7. Masters IB, Chang AB, Patterson L, Wainwright C, Buntain H, Dean BW, Francis PW. Series of laryngomalacia, tracheomalacia, and bronchomalacia disorders and their associations with other conditions in children. Pediatric pulmonology. 2002 Sep;34(3):189-95.

8. Krashin E, Ben-Ari J, Springer C, DeRowe A, Avital A, Sivan Y. Synchronous airway lesions in laryngomalacia. International journal of pediatric otorhinolaryngology. 2008 Apr 1;72(4):501-7.

9. Thompson DM. Abnormal sensorimotor integrative function of the larynx in congenital laryngomalacia: a new theory of etiology. The Laryngoscope. 2007 Jun;117(S114):1-33.

10. Hartl TT, Chadha NK. A systematic review of laryngomalacia and acid reflux. Otolaryngology-Head and Neck Surgery. 2012 Oct;147(4):619-26.

11. Martins RHG, Dias NH, Castilho EC et al (2006) Endoscopic findings in children with stridor. Rev Bras Otorrinolaringol 72:649-653

12. Ahmad Latoo M, Jallu AS. Subglottic Stenosis in Children: Preliminary Experience from a Tertiary Care Hospital. International Journal of Otolaryngology. 2020 Dec 15;2020.

13. Monnier P. Acquired post-intubation and tracheostomy-related stenoses. InPediatric airway surgery 
2011 (pp. 183-198). Springer, Berlin, Heidelberg.

14. Yuen HW, Tan HK, Balakrishnan A. Synchronous airway lesions and associated anomalies in children with laryngomalacia evaluated with rigid endoscopy. International journal of pediatric otorhinolaryngology. 2006 Oct 1;70(10):1779-84. 